\title{
Spontaneous intracranial hemorrhage in a patient with Middle East respiratory syndrome corona virus
}

\author{
Fahad M. Al-Hameed, MD, FRCPC.
}

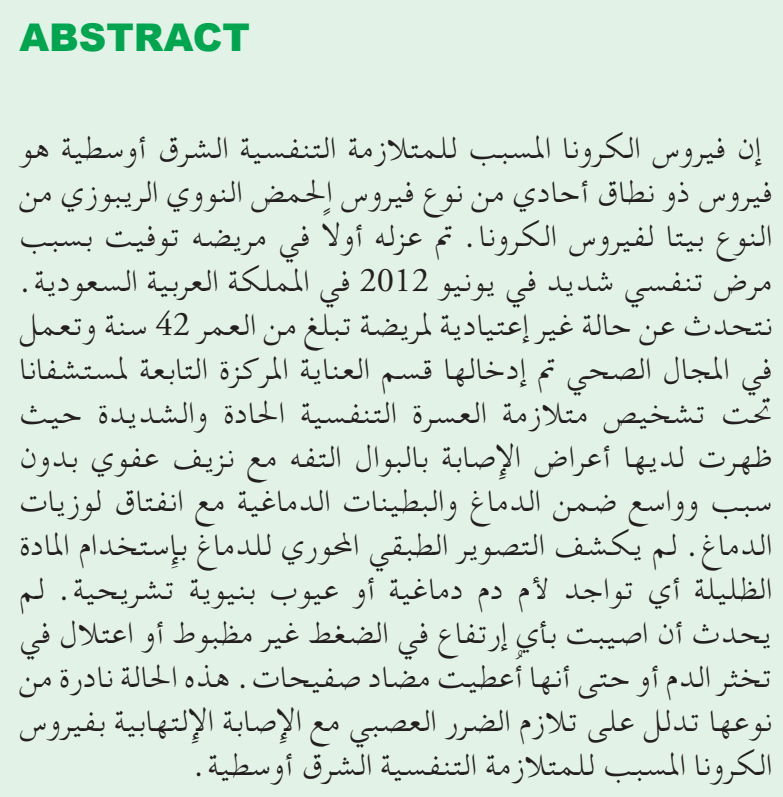

The Middle East respiratory syndrome corona virus (MERS-CoV) is a novel positive sense singlestranded ribonucleic acid virus of the genus Beta corona virus. This virus was first isolated from a patient who died from severe respiratory illness in June 2012 in Jeddah, Kingdom of Saudi Arabia. We describe an unusual case of a 42 year old healthcare worker who was admitted to our Intensive Care Unit (ICU) King Abdul-Aziz Medical City, with MERS-CoV and severe acute respiratory distress Syndrome and developed a sudden-onset diabetes insipidus and spontaneous massive intracranial hemorrhage with intra-ventricular extension and tonsillar herniation. Computed angiogram of the brain did not reveal any aneurysm or structural defects. She never had uncontrolled hypertension, or coagulopathy, nor she received antiplatelets. We are reporting a rare case of structural neurological damage associated with MERS-CoV infection.

Saudi Med J 2017; Vol. 38 (2): 196-200

doi: 10.15537/smj.2017.2.16255
From the Department of Intensive Care, College of Medicine, King Saud bin Abdul-Aziz University for Health Sciences, King Abdul-Aziz Medical City, National Guard Health Affairs, Jeddah, Kingdom of Saudi Arabia

Received 6th September 2016. Accepted 2nd November 2016.

Address correspondence and reprint request to: Dr. Fahad Al-Hameed, Department of Intensive Care, College of Medicine, King Saud bin Abdul-Aziz University for Health Sciences, King Abdul-Aziz Medical City, National Guard Health Affairs, Jeddah, Kingdom of Saudi Arabia. E-mail: fahadalhameed@hotmail.com

ORCID: http://orcid.org/0000-0003-0231-871X

$\mathrm{T}$ The Middle East respiratory syndrome corona virus (MERS-CoV) is a novel positive sense single stranded ribonucleic acid virus of the genus Beta corona virus. ${ }^{1}$ The virus was first isolated from a patient who died from severe respiratory illness in June 2012 in King Abdul-Aziz Medical City, Jeddah, Kingdom of Saudi Arabia. ${ }^{2}$ As of May 25, 2016, the World Health Organization reported 1733 laboratory-confirmed cases of the Middle East respiratory syndrome (MERS) in 27 countries (almost 80\% arising from the Kingdom of Saudi Arabia [KSA]), with an overall mortality rate of $36 \% .^{3}$ The spectrum of the clinical presentation of MERS-CoV patients is wide, and it ranges from asymptomatic infection, mild to severe pneumonia that leads to respiratory failure, septic shock, multiorgan failure, and death. Most of the patients present with fever (98\%), fever with cough (83\%), and difficulty of breathing (72\%). ${ }^{4}$ The main system that is affected by MERS-CoV is the upper airway tract followed by the lower respiratory tract system. However, MERS-CoV rarely affects the central nervous system. We describe a case of a primary care physician who suffered from a MERS-CoV infection and during her hospitalization course she developed a suddenonset of diabetes insipidus and massive spontaneous intracranial hemorrhage $(\mathrm{ICH})$ that was complicated with intra-ventricular extension and tonsillar herniation leading to death. We are reporting an unusual case of direct structural neurological injury associated with MERS-CoV infection. Informed consent was officially given by the husband. 
Case Report. A-42-year old female health care provider presented to the emergency department with high-grade fever, productive cough, shortness of breath, and bony pains of 2 days duration. She had a positive history of contact with a patient of similar complaints 2 days earlier to her presentation. Her past medical history was relevant for left nephrectomy in 2012 for staghorn calculus, caesarian section 3 times, and abortion once. She was obese and had been newly diagnosed with diabetes mellitus type 2 however, she was not on any diabetic medication. On presentation, her Glasgow Coma Scale (GCS) was 15/15, she was hemodynamically stable and her laboratory work-up was normal except for leukopenia with a white cell count of 3.7 and lymphopenia $0.39 \%$. Her chest $\mathrm{x}$-ray showed bilateral infiltrates, which were more prominent on the right side (Figure 1). The patient was admitted to the ward after the initial septic screen was taken for MERS-CoV, influenza $A B, H 1 N 1$ (swine flu), dengue serology, and malaria tests. She was started on broad spectrum antibiotics and oseltamivir empirically. All her previous tests were negative. As her symptoms progressed in the medical ward, she was admitted to the Intensive Care Unit (ICU). Her chest $\mathrm{x}$-ray on the day of admission to the ICU, showed extensive bilateral consolidations (Figure 2). Due to her refractory hypoxaemia, she was electively intubated and mechanically ventilated requiring $100 \%$ Fraction of inspired oxygen for which she was started on the acute respiratory distress syndrome (ARDS) protocol which included lung protective strategies with low tidal volume as well as prone position. Her tracheal aspirates was sent again for MERS-CoV virus, using real time polymerase chain reaction assay (Roche, Berline, Germany) and the result came back positive. She was started on Peginterferon Alpha-2a, ribavirin, and intravenous methylprednisolone $60 \mathrm{mg}$ every 6 hours along with other supportive measures. At some point during her initial course in the ICU, Extracorporeal membrane oxygenation treatment was contemplated. However, on day 5 of ICU admission, she showed a fair improvement in her respiratory function. On day 12 , she was on $40 \%$ FiO2 with a Partial Pressure of Oxygen in arterial blood $=103.5 \mathrm{~mm} \mathrm{Hg}$. A weaning trial was planned with sedation cessation, methylprednisolone tapered to $40 \mathrm{mg}$ IV every 6 hours and Peginterferon Alpha-2a and ribavirin were discontinued. She remained

Disclosure. Authors have no conflict of interest, and the work was not supported or funded by any drug company. hemodynamically stable, her respiratory function and radiological features showed significant improvement on her chest consolidation (Figure 3) and she started to wake up and move all her limbs. On day 13, the patient progressively became polyuric with a urine osmolarity of 95 , serum osmolarity of 341 , urine sodium less than 20, serum sodium $161 \mathrm{meq} / \mathrm{L}$, chloride $119 \mathrm{meq} / \mathrm{L}$, and blood sugar $25 \mathrm{mmol} / \mathrm{L}$. Patient was managed with Desmopressin 2 ug subcutaneously with the plan to do brain computed tomography. However, on the same night, she suddenly became unresponsive, her GCS dropped to $3 / 15$, and her pupils were $3 \mathrm{~mm}$ wide with sluggish reaction. Urgent CT brain showed right frontal hematoma, subarachnoid hemorrhage extending to ventricles, causing midline shift and

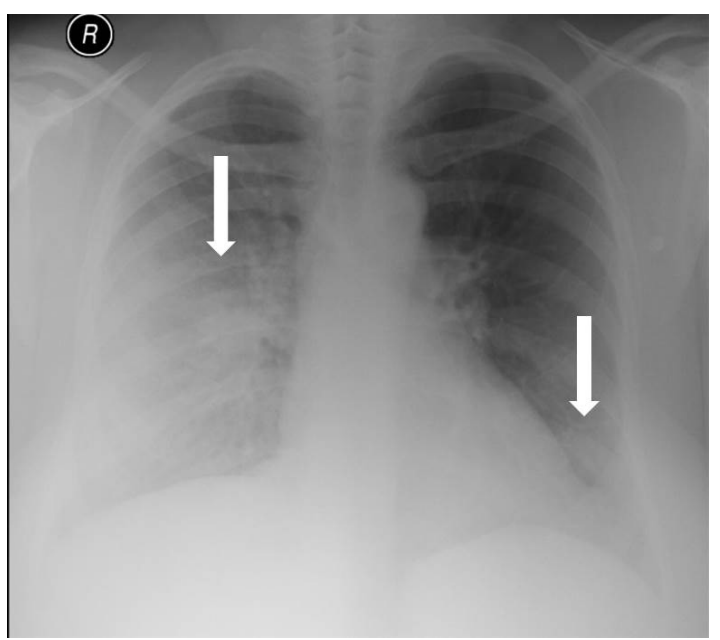

Figure 1 - Chest $\mathrm{x}$-ray showed bilateral infiltrates which were more prominent on the right side (arrows).

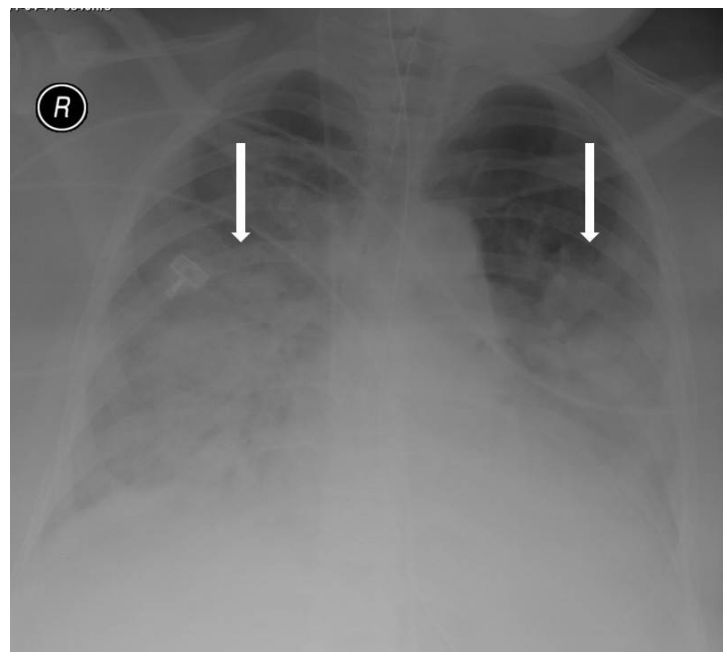

Figure 2 - Chest x-ray on the day of admission to the intensive care unit, showed extensive bilateral consolidations (arrows). 
subfalcine herniation (Figure $4 \& 5$ ). She had a normal platelet count of 347 X 109 , as well as a normal coagulation profile that day. She was not on any form of anti-coagulation treatment. Furthermore, all her blood cultures were negative. She was reviewed by the neurologists and neurosurgical team, their opinion were that the intracranial bleed was acute and large, and their plan was for no surgical intervention and advised only for medical supportive measures. She clinically

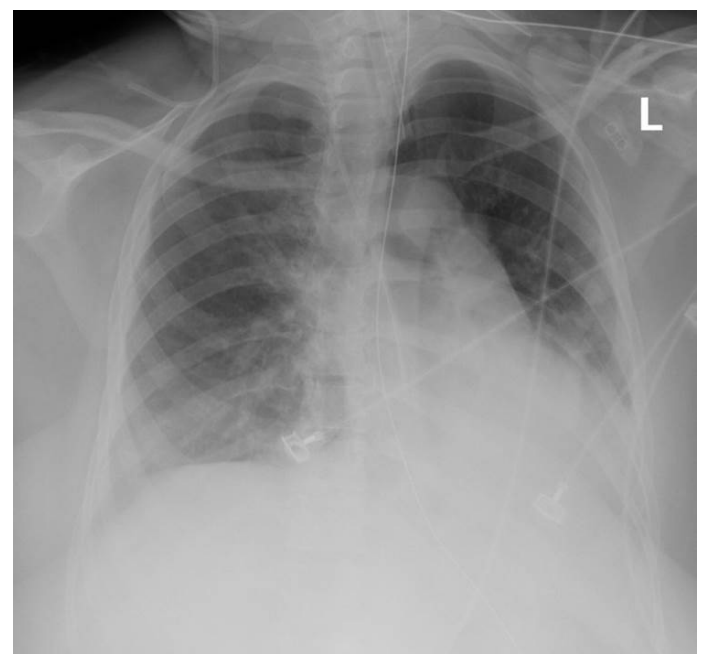

Figure 3 - Chest x-ray showed significant improvement on her chest consolidation.

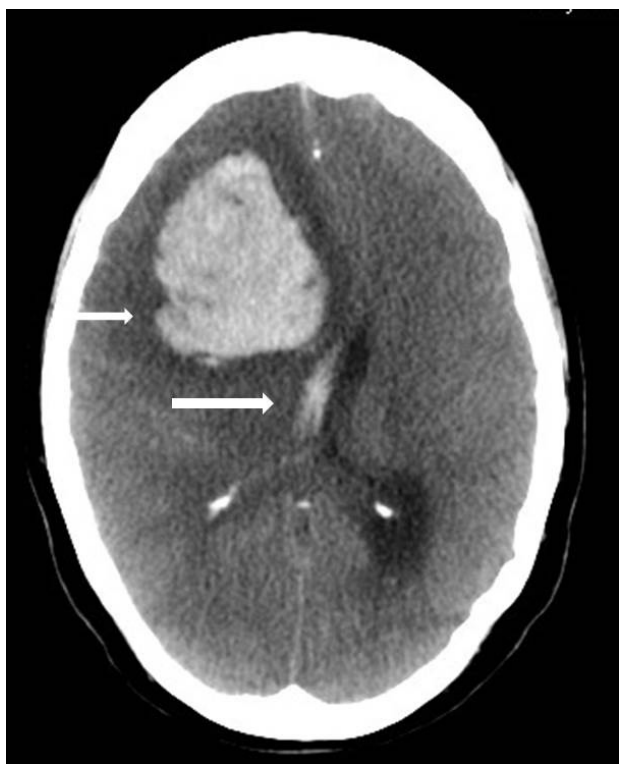

Figure 4 - Computed tomography brain showed right frontal hematoma, subarachnoid hemorrhage extending to ventricles, causing midline shift and subfalcine herniation (arrows). lost all her brain stem reflexes and her pupils became fixed and dilated. A follow up brain CT scan with contrast showed complete loss of gray and white matter differentiation of both cerebral hemispheres with large frontal hematoma, and complete effacement of extra axial CSF spaces including lateral ventricles and basal cisterns (Figure 6). The contrast enhanced CT images showed no enhancement of intracranial vessels beyond the intracranial supraclinoid segments of the internal

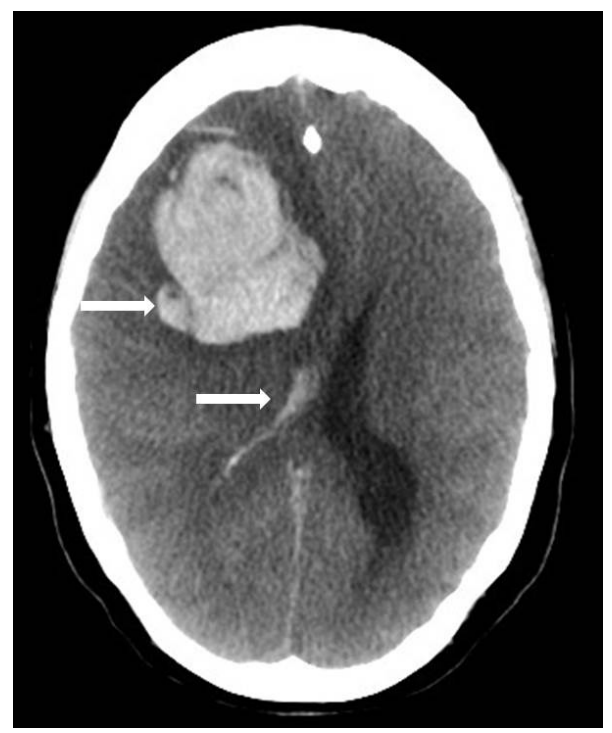

Figure 5 - Computed tomography brain showed right frontal hematoma, subarachnoid hemorrhage extending to ventricles, causing midline shift and subfalcine herniation (arrows).

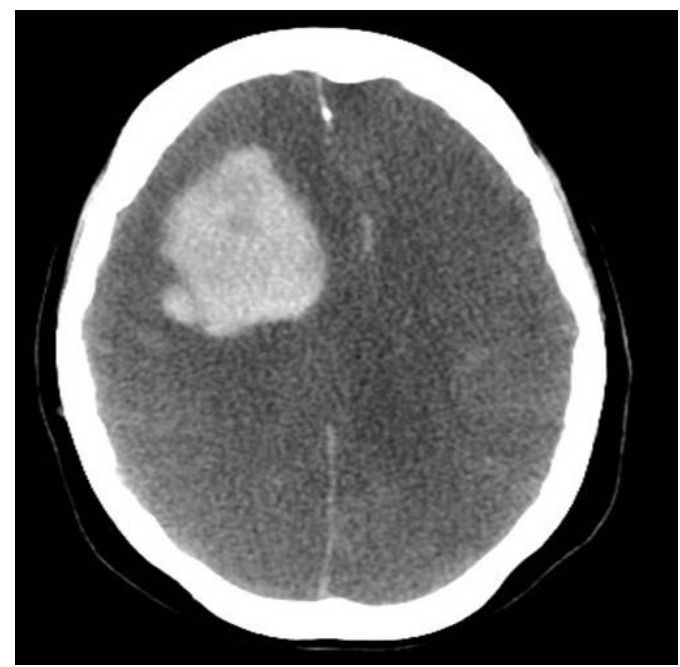

Figure 6 - Brain computed tomography scan with contrast showed complete loss of gray and white matter differentiation of both cerebral hemispheres with large frontal hematoma, and complete effacement of extra axial cerebrospinal fluid spaces including lateral ventricles and basal cisterns. 
carotid arteries. Computed tomography angiogram revealed no visualization of Middle cerebral artery, post cerebral artery and Anterior communicating arteries, and no flow within the posterior circulation. The findings of complete brain anoxia and lack of intracranial flow were consistent with brain death criteria. Due to a family psychological trauma and social issues, all her supportive measures continued for some time. On day 111 of ICU admission, she had a cardiac arrest and was declared dead on the same day.

Discussion. The Middle East respiratory syndrome corona virus infection typically begins with fever, cough, dyspnea, and that rapidly progress to pneumonia requiring ventilatory and other organ support. Risk factors for the development of severe disease in addition to co-morbidities and an immunocompromised state include obesity, diabetes, and cardiopulmonary diseases. Concomitant infections and low albumin levels are predictors of severe illness. An age above 65 in a case series was observed to have high mortality. 4,5 Our patient was a young primary care physician who probably got exposed to a patient with MERS-CoV infection and developed severe disease. She was about to recover but unfortunately she suddenly developed new onset diabetes insipidus and spontaneous ICH, which raised many unanswered clinical questions. The incidence of sudden onset of spontaneous $\mathrm{ICH}$ could not be pathologically explained in the absence of risk factors, such as uncontrolled hypertension, coagulopathy, antiplatelet medications, and cerebral aneurysms or structural abnormality. Her risk factors, include moderate obesity (99 kg), Body mass index of 35, newly diagnosed diabetes, left nephrectomy for staghorn calculus with normal renal function and obstetric history of abortion once and cesarean section 3 times. An interesting observation of severity of MERS in obese patients can be explained as dipeptidyl peptidase 4 (DPP4) also known as (CD26) an enzyme involved in glucose metabolism expressed on the surface of most cell types for example, T cells, lung, kidney, placenta, liver, pancreas, heart, and brain. It is expressed in large numbers in obese people compared with non-obese individuals. The DPP4 has also been shown to be the port of entry of MERS-CoV into human cells. ${ }^{6,78}$ Coincidentally, on the day she developed spontaneous intraventricular hemorrhage her blood sugar level was $25 \mathrm{mmol} / \mathrm{L}$, which may be a modifiable risk factor and needs emphasis on the strict glycaemic control in the management of MERS-CoV patients especially those that are obese which needs further study to know the pathogenesis in understanding the outcomes.
As in our case, with diabetes insipidus, the possibility of altered fluid and electrolyte homeostasis can not be ruled out with MERS-CoV infection. One of the human corona viruses is MERS-CoV that include other strains like severe acute respiratory syndrome associated coronavirus (SARS-CoV). Gu J et al, ${ }^{9}$ reported 8 confirmed cases of brain necropsy of SARS-CoV that was found to have a direct viral infection of neuronal cells located in the cortex and hypothalamus with edema suggesting neuronal ischemia type of injury. $\mathrm{Xu} \mathrm{J}$ et al ${ }^{10}$ also confirmed the direct capability of SARS-CoV of infecting the brain and central nervous system using transmission electronic microscopy and nested reverse transcription-polymerase chain reaction (PCR). In our patient, diabetes insipidus and spontaneous $\mathrm{ICH}$ occurred after discontinuing the Peginterferon Alpha-2a and ribavirin therapy, that raises an interesting question regarding the duration of antiviral therapy in the absence of specific guidelines. ${ }^{10,11}$ Middle East respiratory syndrome corona virus PCR remained positive in this patient even after one month period, this raises the question of whether the viral load and severity of infection including systemic spread to other organ systems needs to be studied in detail. Serial sampling of PCR testing from the upper, lower respiratory tracts and other body parts for example, serum, urine, stool, and cerebral spinal fluids are recommended to understand the viral replication kinetics and guide the infection control. As no CSF sample of MERS-CoV could be observed in this case due to tonsillar herniation, a possibility that could not be excluded as previously reported cases of MERS-CoV in CSF indicates the penetration of neuronal and glial cells by MERS-CoV infection. Three cases of severe neurological syndrome associated with MERS-CoV infection have been reported recently by Arabi, et al, ${ }^{12}$ but all presented with central nervous system manifestations and had significant MRI findings however, none of them developed intracerebral hemorrhage like in our case.

In conclusion, MERS-CoV infected patients who develop critical illness often present with severe ARDS, multiorgan failure, septic shock, and encephalopathy. Since 4 years of the initial report of MERS-CoV infection, the pathophysiology of multiorgan failure, septic shock is still not understood well. Although it is less commonly involved, central nervous system can be affected either functionally or structurally by MERS-CoV infection. Appropriate management with multidisciplinary care is recommended to control and manage the viral infection which is emerging with new global challenges. 
Acknowledgment. The author gratefully acknowledge Dr. Abdulrahman AlShami, Departemnt of Radiology, Dr. Ghulam Rasool and Mrs. Ohoud AlOrabi, Department of Intensive Care, King Abdul-Aziz Medical City, Jeddah, Kingdom of Saudi Arabia.

\section{References}

1. Van Boheemen S, de Graaf M, Lauber C, Bestebroer TM, Raj VS, Zaki AM, et al. Genomic characterization of a newly discovered coronavirus associated with acute respiratory distress syndrome in humans. MBio 2012; 3: 473-412.

2. Zaki AM, van Boheemen S, Bestebroer TM, Osterhaus AD, Fouchier RA. Isolation of a novel coronavirus from a man with pneumonia in Saudi Arabia. N Engl J Med 2012 8; 367: 18141820 .

3. World Health Orgnization: Middle East respiratory syndrome coronavirus (MERS-CoV). WHO. Geneva (CH); 2015 Avaliable from: http://www.who.int/emergencies/mers-cov/en/

4. Al-Tawfiq JA, Assiri A, Memish ZA. Middle East respiratory syndrome novel corona MERS-CoV infection. Epidemiology and outcome update. Saudi Med J 2013; 34: 991-994.

5. Arabi YM, Arifi AA, Balkhy HH, Najm H, Aldawood AS, Ghabashi A, et al. Clinical course and outcomes of critically ill patients with Middle East respiratory syndrome corona virus infection. Ann Intern Med 2014; 160: 389-397.
6. Raj VS, Mou H, Smits SL, Dekkers DH, Müller MA, Dijkman $\mathrm{R}$, et al. Dipeptidyl peptidase 4 is a functional receptor for the emerging human corona virus-EMC. Nature 2013; 495: 251-254.

7. Lamers D, Famulla S, Wronkowitz N, Hartwig S, Lehr S, Ouwens DM, et al. Dipeptidyl peptidase 4 is a novel adipokine ptenially linking obesity to the metabolic syndrome. Diabetes 2011; 60: 1917-1925.

8. Barnett A. DPP-4 inhibitors and their potential role in the management of type 2 diabetes. Int J Clin Pract 2006; 60: 1454-1470.

9. Gu J, Gong E, Zhang B, Zheng J, Gao Z, Zhong Y, et al. Multiple organ infection and the pathogenesis of SARS. J Exp Med 2005; 202: 415-24.

10. Xu J, Zhong S, Liue J, Li L, Li Y, Wu X, et al. Detection of severe acute respiratory syndrome corona virus in the brain: potential role of the chemoking mig in pathogenesis. Clin Infect Dis 2005; 41: 1089-1096.

11. Falzarano D, de Wit E, Rasmussen AL, Feldmann F, Okumura A, Scott DP, et al. Treatment with interferon-alph2b and ribavirin improves outcome in MERS-CoV-infected rhesus macaques. Nat Med 2013; 19: 1313-1317.

12. Arabi YM, Harthi A, Hussein J, Bouchama A, Johani S, Hajeer $\mathrm{AH}$, et al. severe neurologic syndrome associated with Middle East respiratory syndrome corona virus (MERS-CoV). Infection 2015; 43: 495-501.

\section{Case Reports}

Case reports will only be considered for unusual topics that add something new to the literature. All Case Reports should include at least one figure. Written informed consent for publication must accompany any photograph in which the subject can be identified. Figures should be submitted with a $300 \mathrm{dpi}$ resolution when submitting electronically. The abstract should be unstructured, and the introductory section should always include the objective and reason why the author is presenting this particular case. References should be up to date, preferably not exceeding 15 . 